\title{
Contemporary Strategies in the Management of Hepatocellular Carcinoma
}

\author{
Shirin Elizabeth Khorsandi and Nigel Heaton \\ Institute of Liver Studies, King's College Hospital, Denmark Hill, London SE5 9RS, UK \\ Correspondence should be addressed to Shirin Elizabeth Khorsandi, s.khorsandi@imperial.ac.uk
}

Received 12 July 2012; Accepted 17 September 2012

Academic Editor: Andrea Lauterio

Copyright ( $) 2012$ S. E. Khorsandi and N. Heaton. This is an open access article distributed under the Creative Commons Attribution License, which permits unrestricted use, distribution, and reproduction in any medium, provided the original work is properly cited.

\begin{abstract}
Liver transplantation is the treatment of choice for selected patients with hepatocellular carcinoma (HCC) on a background of chronic liver disease. Liver resection or locoregional ablative therapies may be indicated for patients with preserved synthetic function without significant portal hypertension. Milan criteria were introduced to select suitable patients for liver transplant with low risk of tumor recurrence and 5-year survival in excess of 70\%. Currently the incidence of HCC is climbing rapidly and in a current climate of organ shortage has led to the re-evaluation of locoregional therapies and resectional surgery to manage the case load. The introduction of biological therapies has had a new dimension to care, adding to the complexities of multidisciplinary team working in the management of HCC. The aim of this paper is to give a brief overview of present day management strategies and decision making.
\end{abstract}

\section{Introduction}

Hepatocellular carcinoma (HCC) is the fifth most common cancer in the world. Ninety percent of primary liver cancers are HCC, the majority of which develop on the background of cirrhosis. Over the past decade, medical management of the patient with chronic liver disease has improved. In parallel, the prevalence of hepatitis $\mathrm{B}(\mathrm{HBV})$, hepatitis $\mathrm{C}$ virus (HCV), alcohol related liver disease, and NASH has increased and combined with an ageing population has led to a surge in the number of cases worldwide [1-3]. As a consequence, HCC is an important complication of cirrhosis and a leading indication for liver transplantation (LT), accounting for approximately a third of patients on transplant waiting lists [4]. The introduction of surveillance using alphafetoprotein and ultrasound has led to the earlier recognition of HCC and increases the therapeutic options available [5]. In the absence of treatment the overall 5year survival is $<10 \%$ [6]. These include LT, resection, locoregional, and systemic therapies. For a solitary HCC with preserved liver function and low hepatic vein pressure gradient, liver resection still remains the first choice.
Historically, survival rates were $35-62 \%$ at 3 years and $17-50 \%$ at 5 years for patients with cirrhosis undergoing resection for HCC $[6,7]$. However, tumor recurrence rates were high, up to $70 \%$, and progression to liver failure was common $[6,8-10]$. LT is an attractive treatment option as it treats both the cancer and underlying liver disease. In the 1980s, patients presenting with large HCC were considered good candidates for LT as they were in better condition than patients with chronic liver disease and were more likely to survive the perioperative period but they had large or multifocal tumors. This resulted in a high recurrence rate of up to $65 \%$, a 5 year survival of $10-35 \%$, and a median survival of those with recurrence of 6 months, coupled with increasing demand for donor livers led to a more restrictive selection process [11-13]. Recognition that small tumors appeared to fare better after LT led Mazzaferro to introduce the Milan criteria to select patients leading to improved survival with low rates of tumor recurrence [14]. By adhering to the Milan criteria of undertaking LT for HCC with a solitary tumor $\leq 5 \mathrm{~cm}$ or 3 tumors $\leq 3 \mathrm{~cm}$ each, a 5 year survival greater than $70 \%$ and recurrence rates of $<10 \%$ were produced. 


\section{Staging}

A staging system for HCC poses problems because the presence of liver disease and tumor varies due to the different epidemiological backgrounds and risk factors. The ideal staging system needs to include prognostic information regarding both the cancer and liver functional status and take account of clinical factors that influence response to treatment. The TNM classification is an oncology standard useful in conjunction with the presence of microvascular invasion of examined resection or explanted tumors/liver and provides information regarding the risk of tumor recurrence but does not take account of the liver functional status. As TNM requires pathological data (microvascular invasion) and only 20\% HCC are resected for which it is good at discriminating stages for, its usability is limited. The Okuda staging system has been widely used since 1985. It uses four criteria of ascites, albumin, bilirubin, and tumor size to assess liver functional status and tumor stage. It is a good system for stratifying advanced/symptomatic disease but less useful in early stage to guide treatment choices. Other available systems are the French classification, the Cancer of the Liver Italian Program (CLIP) classification, and the Barcelona-Clínic Liver Cancer (BCLC) staging system; the Chinese University Prognostic Index (CUPI score) and the Japan Integrated Staging (JIS) or bm-JIS if biomarkers are included [15]. The CUPI and CLIP scores mainly stratify patients at advanced stages; only two include prognostic variables (BCLC, CUPI) and only one allocates treatment according to specific prognostic subclasses (BCLC). The BCLC is emerging as the standard staging system for HCC in the West and has been externally validated and incorporates prognostic variables related to tumor (size, number, vascular invasion, N1, M1), liver function (Child-Pugh), and health status (ECOG-Eastern Cooperative Oncology Group Performance Status). As well as incorporating variables that influence therapy such as bilirubin, portal hypertension, and presence of symptoms to assist in treatment decision making (see Figure 1).

\section{Liver Transplant}

LT is the treatment of choice for small multifocal $\mathrm{HCC} \leq 3$ tumors and $\leq 3 \mathrm{~cm}$ or a single tumor $\leq 5 \mathrm{~cm}$ with significant liver functional impairment. Better selection of patients has improved 5 year survival to $>70 \%$ and recurrence $<10 \%$. The major limitation to LT as treatment for HCC is the scarcity of cadaveric donors and the associated waiting time that results in a $20 \%$ drop-out rate and potentially increases the risk of recurrence from extension of vascular invasion. The use of tumor size and number to try to reflect tumor biology has been successful. However, it is clear that some patients with favourable "biology" are excluded. A number of groups have tried to expand indications beyond the Milan criteria and claim to achieve similar survival rates $[16,17]$. The University of California San Francisco (UCSF) criteria are probably the best known and include one tumor $\leq 6.5 \mathrm{~cm}$ or multiple tumors of which the largest is $4.5 \mathrm{~cm}$ and the sum of all diameters is $\leq 8 \mathrm{~cm}$ [16]. More recently the up-to-7 criteria, where the HCC scores 7 based on the sum of the largest tumor (diameter $\mathrm{cm}$ ) and the total number of tumors, have been introduced [17]. The majority of the studies supporting extension of the Milan Criteria are based on retrospective histological analysis of the tumor burden in the explant liver and have not been validated prospectively [16-19].

Another area where the principles of the Milan Criteria have been challenged is in salvage LT. Salvage LT has been advocated by some to manage HCC within Milan Criteria after resection [20]. In selected cases, similar overall 5 year survival for salvage LT as primary LT for HCC has been achieved (provided the comparison is from time listed for LT rather than date of LT, that is, intention to treat bias). There is continuing debate regarding whether previous resection compromises the subsequent LT [21]. Other groups have found salvage LT to have a high operative mortality, $23.5 \%$ versus $2.1 \%$ for primary LT, higher recurrence rates, and poorer overall 5 year survival of $41 \%$ [22]. Salvage LT remains controversial at a time of a limited resource with tumor characteristics, background liver (cirrhotic or noncirrhotic), and centre experience appearing to be the main determinants of recurrence and survival.

An alternative strategy to expanding the criteria for LT is to downstage to within Milan Criteria aiming to achieve patient survival and recurrence free survival rates similar to those treated at an earlier stage. This is distinct from bridging therapy. Bridging therapy is utilised to maintain the tumor within listing criteria while a suitable graft is awaited for on the waiting list. Bridging therapy is a widely accepted practice whereas downstaging for LT is not $[23,24]$. To be eligible for downstaging locoregional therapy, there should be no radiological evidence of vascular invasion. There is no consensus limit to tumor number or size [25]. Predictors of downstaging failure are tumors with an infiltrative pattern [26] and an AFP > $1000 \mathrm{ng} / \mathrm{mL}$ [27]. There is evidence to suggest that downstaging of HCC to within Milan Criteria can produce reasonable results [25, 27]. But the data is difficult to interpret as the studies utilise different inclusion criteria (tumor size and number), locoregional therapies either individually or in combination, and endpoints. Current published data reveal that after downstaging, surgical resection rates vary widely between $7 \%$ and $18 \%$ producing 5 year survival rates of between $25 \%$ and 57\% [28] and LT rates range between 24\% and 90\%, with an intention to treat post HCC treatment survival of between $60 \%$ and $70 \%$ at 3 years $[27,29,30]$.

Living donor LT (LDLT) is a good graft option for HCC as it allows neoadjuvant treatment to be organized around a LT. It provides a high-quality graft and removes a competing HCC recipient from the waiting list. But higher recurrence rates and reduced survival have been reported when compared to cadaveric LT [31-33]. Explanations for this observation include growth factors released from the regenerating liver may stimulate cancer cell growth. The shorter waiting time for LDLT may remove the observation period that occurs on the waiting list to assess tumor biology and a 3 month cooling off period has been advocated before undertaking LDLT. Surgical oncological clearance may also be compromised as the IVC has to be preserved for LDLT 


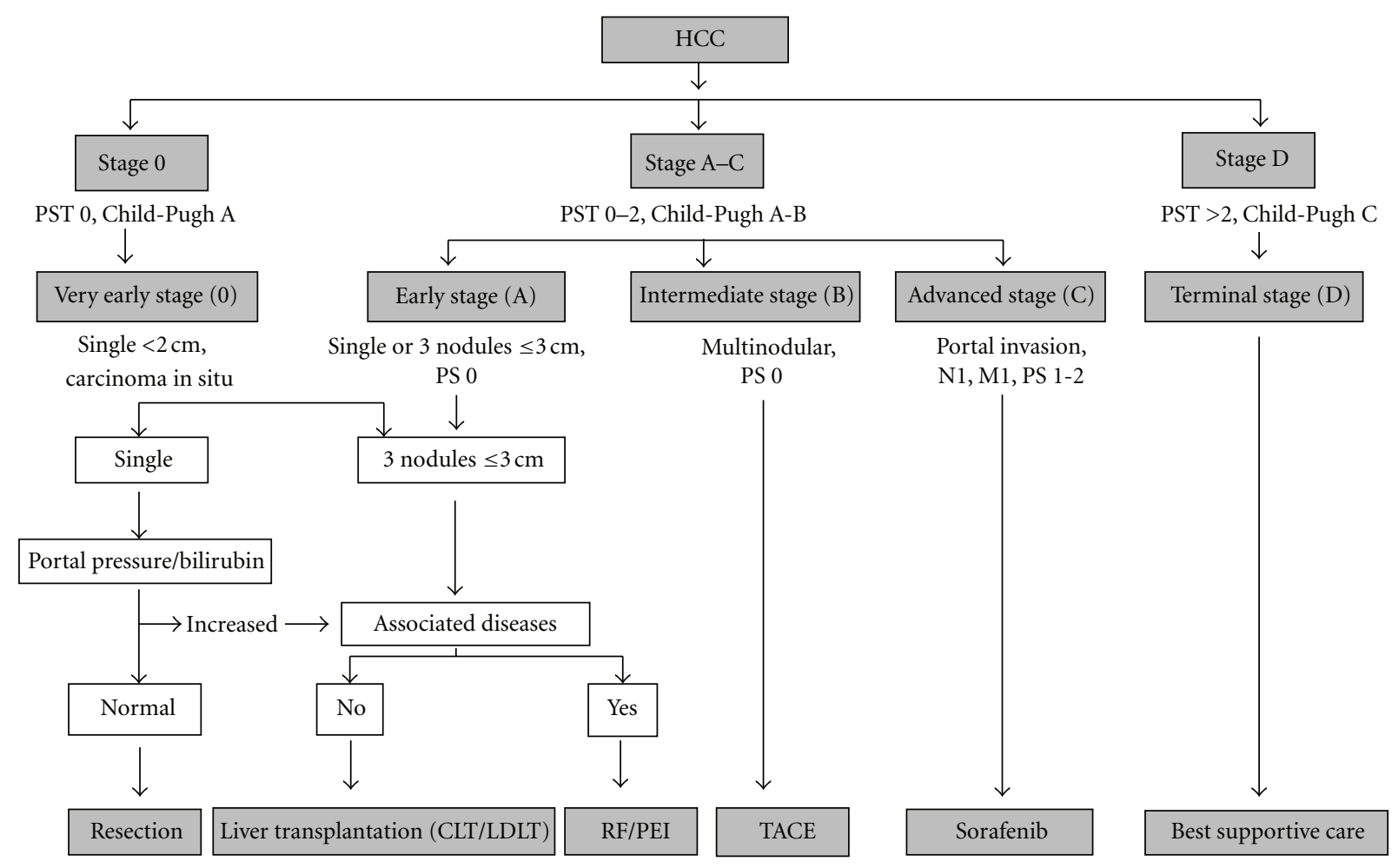

FIgure 1: The Barcelona-Clínic Liver Cancer (BCLC) staging system for HCC. M: metastasis classification; N: node classification; PST: performance status; RFA: radiofrequency ablation; TACE: transarterial chemoembolization.

[34]. In addition, an element of institutional bias may lead to LDLT in HCC with a higher risk of recurrence. On multivariate analysis of published studies on LDLT versus cadaveric LT, graft type, and waiting time have not been found to be significant risk factors for recurrence post LT. If LDLT is undertaken for HCC outside regional criteria and the graft fails retransplantation with cadaveric LT is ethically contentious [35]. The donor risk and the degree of benefit to the recipient needed to justify LDLT for advanced HCC are still undetermined and for now many centres have adopted the same criteria/therapeutic goals for LDLT as cadaveric LT [36].

\section{Liver Resection}

Resection is the treatment of choice in noncirrhotics. Noncirrhotic HCC accounts for $5 \%$ of cases in the West and $40 \%$ of cases in Asia. Patients with cirrhosis suitable for resection need preserved liver function and a hepatic venous pressure gradient $\leq 10 \mathrm{mmHg}$. Anatomic resection is advocated by some as being more preferable to nonanatomic, as it is thought to produce better outcome by eliminating intrahepatic metastases in the related portal vein tributary [37]. In patients with cirrhosis selected on liver functional status, the main predictors for survival are tumor size, multiplicity, and vascular invasion. Five-year survival for tumors $\leq 2 \mathrm{~cm}, 2-5 \mathrm{~cm}$, and $>5 \mathrm{~cm}$ are $66 \%, 52 \%$, and $37 \%$, respectively. For single tumors the 5 year survival is $57 \%$ and for multiple $26 \%$ but some centres are achieving $>50 \%$ in multiple HCC within the Milan Criteria but otherwise are not suitable for LT [38]. Recurrence remains problematic occurring in $70 \%$ at 5 years; true recurrence/intrahepatic metastases generally occur within 2 years of resection; if greater than 2 years it is generally regarded as a de novo tumor or late recurrence [39]. At present there is no evidence that neoadjuvant/adjuvant therapy has any efficacy in reducing recurrence after resection [40]. Downstaging locoregional therapy can be employed to facilitate resection in disease which is initially regarded as unresectable and can achieve reasonable outcome, with 5 year survivals of 25-67\%, with the possibility of cure [28]. Preoperative portal vein embolisation can be employed to increase future remnant liver volume to allow more extensive resections to be undertaken but the complication rate in cirrhotics is 10 $20 \%$ and its effectiveness in this patient group is not fully established [41]. A laparoscopic approach to resection in cirrhotics has been proposed by some to reduce the operative insult and the risk of decompensation [42].

\section{Locoregional Therapy: Ablation, TACE, and Radiation}

There are a number of different locoregional strategies available or being developed but the largest experience is with transarterial chemoembolization (TACE) and radiofrequency ablation (RFA). Percutaneous ethanol injection (PEI) was the first chemical ablative technique utilised. When applied to small tumors $<2 \mathrm{~cm}$ PEI produces $90 \%$ necroses and a 5 -year survival of $47-53 \%$ but is limited by high 
recurrence rates of approximately $40 \%$. Chemical ablation has now been superseded by thermal techniques such as radiofrequency ablation (RFA). RFA is the most well-studied alternative to PEI producing better local tumor control with a 2 year recurrence of $2-18 \%$ and a 5 year survival of $40-70 \%$ or better when the treatment groups have been selected [43]. Meta-analyses of randomised control trials have confirmed that RFA is a more effective way to obtain local tumor control and survival benefit compared to PEI, establishing it as a standard locoregional treatment $[44,45]$.

RFA can be performed percutaneously, laparoscopically, or at open surgery depending on tumor location $[46,47]$. RFA is effective for early small $\mathrm{HCC}<3 \mathrm{~cm}$ when resection or LT are not feasible [48-51], whereas larger tumors may be inadequately treated. Overall $10-25 \%$ of tumors will not be suitable for RFA because of location such as subcapsular, adjacent to the gall bladder or major vessels which increases the risk of complication and inadequate ablation because of heat sink. The recurrence rate after RFA for selected early small HCC can be comparable to that of surgery $[50,51]$. In highly selected HCC $<2 \mathrm{~cm}$ RFA has the potential to be curative with a rate of complete response approaching $97 \%$ and a 5 year survival of $68 \%$. However, randomised control trials of RFA against resection for small $\mathrm{HCC}<3 \mathrm{~cm}$ have failed to show that RFA is as effective as resection but the majority of studies were underpowered or had incomplete follow up [49, 52]. Increasingly, RFA is being considered as an alternative initial "curative" treatment option for small centrally placed HCC as it offers the advantages of preserving parenchyma, potentially removes competition from the transplant waiting list and based on location, effective tumor necrosis can be obtained [43, 48].

Solitary HCC $>3 \mathrm{~cm}$ but $<5 \mathrm{~cm}$ RFA becomes less effective. But when TACE followed by RFA for this size tumor is applied, the therapeutic effect of RFA is significantly increased and reduces tumor progression rate to $6 \%$ compared to $39 \%$ for RFA alone [53]. In larger HCC $>5 \mathrm{~cm}$ outside LT criteria or not suitable for resection, ablative strategies may not work in a predictable manner. TACE also has inconsistent results and no advantage has been demonstrated by combining therapies [54]. When RFA is not suitable either because of tumor location or size, novel thermal or nonthermal ablative techniques may overcome the limits of RFA. Promising thermal ablative strategies include microwave producing large areas of ablation with less heat sink and high intensity focused US (HIFU) that can be used in patients with ascites. Alternative non thermal ablative techniques of interest include irreversible electroporation (IE). Ablative technology is improving and further experience will determine its applicability. In assessing the effectiveness of ablation radiologically, the widely used RECIST (the response evaluation criteria in solid tumours) has limitations as it includes both necrotic and viable tumor areas [55] and the modified RECIST that includes the assessment of viable tumor showing uptake in the arterial phase is more reliable.

Based on a meta-analysis TACE is emerging as the standard of care for asymptomatic HCC outside Milan criteria [56], demonstrating improved survival compared to best supportive care. A partial response of $15-55 \%$ can be observed producing a survival benefit, increasing median survival time from 16 months to 20 months with $49 \%$ survival at 2 years [56]. But individually the studies did not clearly demonstrate a benefit, mainly because of heterogenous patient study groups and varying TACE techniques. This implies that good results with TACE are achieved when it is used on a selective basis. Generally TACE is not suitable in decompensated liver disease where there is ascites or jaundice to the avoid major complications and minimize treatment related deaths to less than $2 \%$ [57]. For optimal results TACE needs to be as selective as possible, producing sustained and high localised concentrations within the tumor minimizing systemic exposure. Alternative ways to be delivering chemotherapy instead of the standard ethiodized oil (lipiodol) suspensions are drug-eluting beads [58]. In the PRECISION V trial [59], a randomized control trial, comparing drug-eluting beads with doxorubicin to conventional TACE with doxorubicin found it was better tolerated, with reduced liver toxicity and improved treatment response. Owing to the improved safety and tolerance drugeluting beads could be applicable in higher risk patient groups. Further ways of optimising the therapeutic benefit of TACE is by combining with systemic drugs. Using agents that target the angiogenic pathways that are switched on by the local hypoxia produced by TACE is being evaluated [45]. Generally, if there is no response after two TACE sessions, alternative treatment strategies should be considered, which in the majority will be systemic therapy. In highly selected patients consideration should be given to combination of treatments such as ablation/radioembolization.

Advanced HCC that is symptomatic, exhibiting vascular invasion and/or has extrahepatic disease have a short median survival of 6 months with 25\% surviving a year [60]. Systemics are often the only treatment option for palliation but there is a subset that benefit from locoregional therapy such as where vascular invasion is limited to a venous branch receiving intra-arterial therapies such as TACE [61] or radioembolization [62]. Radioembolization using yttrium90 (90Y) labelled microspheres a beta emitter, appears promising, and may also be effective as a precursor to radical therapy with outcomes similar to TACE $[30,63]$ and Sorafenib [64]. There is a need to be aware of intestinal and lung shunting which may provoke serious complications. There is a minimal embolic effect so when there is main portal vein involvement and TACE is contraindicated radioembolization with yttrium may be a good option. In the absence of portal vein involvement radioembolization in Child A survival is 15.5 months, Child B is 13 months, with a portal vein involvement survival of both Child A + B being 5.6 months [65] with $25-50 \%$ response rates [64].

Cyberknife is a new stereotactic body radiation therapy (SBRT) or stereotactic ablative radiotherapy (SABR) in combination with a robotic system that tracks the tumor during respiration and is able to deliver high dose radiation accurately sparing adjacent normal tissue in a small number of fractions. A number of studies in HCC not suitable for standard locoregional treatment or resection have reported promising results. In $\mathrm{HCC}<100 \mathrm{ml}$ progression free survival 
rates at 6 months, 1 year, and 3 years of $83 \%, 72 \%$, and $68 \%$ respectively, with overall survival at 1 year, and 3 years of $92.9 \%$ and $58.6 \%$ have been reported. It also has utility as local salvage treatment after TACE achieving local control in 95\% [66]. To this date no serious SBRT related toxicities being reported [67-69] but it is not clear whether it can be applied to patients with more severe liver diseases as its threshold for tolerance is not defined.

\section{Systemic Therapies: Antiviral Therapy, Immunosuppression, Biologicals, and Chemotherapy}

Worldwide $78 \%$ of $\mathrm{HCC}$ are viral related with $53 \%$ attributed to $\mathrm{HBV}$ and $25 \%$ to $\mathrm{HCV}$ [70]. Risk of HCC recurrence after treatment is increased with progression of active hepatitis and fibrosis. Antiviral therapy is used as an adjuvant treatment with the aim to reduce viral load and fibrosis with the aim of halting progression of viral induced liver disease and reducing the risk of further HCC developing. Hepatitis $\mathrm{B}(\mathrm{HBV})$ infection increases the risk of HCC recurrence particularly for the patient who is $\mathrm{HBeAg}+$ and/or has a high serum HBV DNA level [71, 72]. Treatment with nucleoside analogues entecavir or tenofovir suppresses HBV DNA levels improving liver function in decompensated liver disease and may reduce the risk of HCC development over time [73]. $\mathrm{HBV}$ antiviral therapy reduces risk of recurrence by $41 \%$ and overall mortality by $73 \%$, mainly because death from liver failure is reduced by $77 \%$ [74]. Use of longterm lamivudine treatment resistance can occur in $70 \%$ over 5 years has given way to alternative nucleoside analogues such as entecavir, telbivudine, and nucleotide analogues such as tenofovir but longterm data is lacking for their effect on reducing HCC recurrence.

Chronic infection with $\mathrm{HCV}$ appears to increase the rate of HCC development in a similar way to HBV. The risk does not change with genotype $(G)$ but a recent metaanalysis suggests that HCV G1b maybe more at risk of HCC transformation [75]. Meta-analysis of adjuvant alpha IFN shows reduction in HCC recurrence and mortality in curatively ablated viral hepatitis related HCC. Individually these studies report no effect. Antiviral potency and the ability to produce a sustained viral response (SVR) in $\mathrm{HCV}$ appear to be associated with reducing the risk of HCC recurrence [76]. More longterm data is needed from the newer protease inhibitors (boceprevir, telaprevir) to determine whether the higher SVR they are able to produce translates into lower risk of HCC longterm.

Immunosuppressive agents compound malignant behaviour as immune surveillance for cancer cells is impaired. Mammalian target of rapamycin (mTOR) inhibitors, for example, Sirolimus is an exception to this rule. mTOR is overexpressed in approximately $2 / 3$ of HCC making it an attractive therapeutic target [77]. To establish whether the immunosuppressive regime affects recurrence rates, data from the SiLVER Study is awaited. The SiLVER Study is a randomised multicenter clinical trial comparing Sirolimus containing to a mTOR inhibitor free immunosuppressive regimes. The study consists of a 3-year enrolment period and a 5-year followup. At present, there is little evidence on whether the immunosuppression regime should be completely changed to a mTOR inhibitor or whether these agents should be added to the preexisting immunosuppressive regime when recurrent HCC presents post LT [35, 78, 79]. CNI exposure should be minimised as there is evidence that this reduces the risk of tumor recurrence long term [80].

HCC is an unique chemoresistant tumor and until 2007 no systemic drug was recommended for its management. In the early 1990s, a number of randomised controlled studies assessed the role of adjuvant chemotherapy but no benefit or efficacy was demonstrated. Multiple agents have been assessed but doxorubicin, an anthracycline, has been most rigorously studied $[81,82]$. The main patient groups that should be considered for adjuvant chemotherapy are those being transplanted for extended criteria or have a high risk of recurrence based on the explant pathology. As patients selected for LT should be at low risk of recurrence the majority will not gain any benefit from routine adjuvant chemotherapy.

Since 2007 Sorafenib, an oral tyrosine kinase inhibitor, has become the standard of care. Based upon the SHARP study that demonstrated Sorafenib improved median survival from 7.9 months to 10.7 months and slowed time to progression from 2.8 to 5.5 months [83]. It is well tolerated with diarrhoea in $8-9 \%$ and hand-foot skin reaction in $8-16 \%$, with side effects leading to its discontinuation in $15 \%$. Sorafenib is regarded as the standard therapy for metastatic disease and for HCC progressing despite optimal locoregional therapy [84]. A number of ongoing studies are establishing Sorafenib's adjuvant role in resection, local ablation (Sorafenib as Adjuvant Treatment in the Prevention of Recurrence of Hepatocellular Carcinoma (STORM)), and TACE. Additionally, a phase 1 study is being undertaken in high-risk patients post-LT that on explant are outside Milan criteria with microvascular or macrovascular invasion or histologically poorly differentiated HCC. At present there is no evidence of increased toxicity in $\mathrm{LT}$ recipients and Sorafenib can produce a response based on published case reports $[85,86]$. Other biological agents entering phase 2 or 3 trials for HCC include EGFR (erlotinib) and VEGFR/FGFR (brivanib) tyrosine kinase inhibitors [87].

\section{Conclusions}

Management of HCC continues to evolve and interventional radiology in the form of TACE \pm RFA increasingly dominates management either as a bridge to LT or to downstage facilitating LT or resection. As locoregional therapy technology advances patients that can be considered either for palliation or potential cure will increase. Criteria for LT listing need to become more sophisticated by incorporating tumor biology in decision making, presently inferred from clinical behaviour but in the future by the use of molecular markers. This will facilitate stratification and individualization of HCC treatment. Ultimately, the aim of LT, irrespective of 
disease etiology is to give the maximum benefit from a limited organ pool.

\section{References}

[1] H. B. El-Serag and A. C. Mason, "Rising incidence of hepatocellular carcinoma in the United States," The New England Journal of Medicine, vol. 340, no. 10, pp. 745-750, 1999.

[2] F. X. Bosch, J. Ribes, M. Díaz, and R. Cléries, "Primary liver cancer: worldwide incidence and trends," Gastroenterology, vol. 127, supplement 1, pp. S5-S16, 2004.

[3] H. B. El-Serag and K. L. Rudolph, "Hepatocellular carcinoma: epidemiology and molecular carcinogenesis," Gastroenterology, vol. 132, no. 7, pp. 2557-2576, 2007.

[4] D. Samuel, M. Colombo, H. El-Serag, R. Sobesky, and N. Heaton, "Toward optimizing the indications for orthotopic liver transplantation in hepatocellular carcinoma," Liver Transplantation, vol. 17, supplement 2, pp. S6-S13, 2011.

[5] V. Santi, F. Trevisani, A. Gramenzi et al., "Semiannual surveillance is superior to annual surveillance for the detection of early hepatocellular carcinoma and patient survival," Journal of Hepatology, vol. 53, no. 2, pp. 291-297, 2010.

[6] J. M. Llovet, J. Fuster, and J. Bruix, "Intention-to-treat analysis of surgical treatment for early hepatocellular carcinoma: resection versus transplantation," Hepatology, vol. 30, no. 6, pp. 1434-1440, 1999.

[7] D. Franco, L. Capussotti, C. Smadja et al., "Resection of hepatocellular carcinomas. Reults in 72 European patients with cirrhosis," Gastroenterology, vol. 98, no. 3, pp. 733-738, 1990.

[8] S. Okada, K. Shimada, J. Yamamoto et al., "Predictive factors for postoperative recurrence of hepatocellular carcinoma," Gastroenterology, vol. 106, no. 6, pp. 1618-1624, 1994.

[9] M. Minagawa, M. Makuuchi, T. Takayama, and N. Kokudo, "Selection criteria for repeat hepatectomy in patients with recurrent hepatocellular carcinoma," Annals of Surgery, vol. 238, no. 5, pp. 703-710, 2003.

[10] R. T. P. Poon, S. T. Fan, C. M. Lo, C. L. Liu, and J. Wong, "Intrahepatic recurrence after curative resection of hepatocellular carcinoma: long-term results of treatment and prognostic factors," Annals of Surgery, vol. 229, no. 2, pp. 216222, 1999.

[11] J. G. O'Grady, R. J. Polson, K. Rolles, R. Y. Calne, and R. Williams, "Liver transplantation for malignant disease," Annals of Surgery, vol. 207, no. 4, pp. 373-379, 1988.

[12] B. Ringe, R. Pichlmayr, C. Wittekind, and G. Tusch, "Surgical treatment of hepatocellular carcinoma: experience with liver resection and transplantation in 198 patients," World Journal of Surgery, vol. 15, no. 2, pp. 270-285, 1991.

[13] P. Moreno, E. Jaurrieta, J. Figueras et al., "Orthotopic liver transplantation: treatment of choice in cirrhotic patients with hepatocellular carcinoma?" Transplantation Proceedings, vol. 27, no. 4, pp. 2296-2298, 1995.

[14] V. Mazzaferro, E. Regalia, R. Doci et al., "Liver transplantation for the treatment of small hepatocellular carcinomas in patients with cirrhosis," The New England Journal of Medicine, vol. 334, no. 11, pp. 693-699, 1996.

[15] K. M. Olthoff, A. Forner, S. Hübscher, and J. Fung, "What is the best staging system for hepatocellular carcinoma in the setting of liver transplantation?" Liver Transplantation, vol. 17, supplement 2, pp. S26-S33, 2011.
[16] F. Y. Yao, L. Ferrell, N. M. Bass et al., "Liver transplantation for hepatocellular carcinoma: expansion of the tumor size limits does not adversely impact survival," Hepatology, vol. 33, no. 6, pp. 1394-1403, 2001.

[17] V. Mazzaferro, J. M. Llovet, R. Miceli et al., "Predicting survival after liver transplantation in patients with hepatocellular carcinoma beyond the Milan criteria: a retrospective, exploratory analysis," The Lancet Oncology, vol. 10, no. 1, pp. 35-43, 2009.

[18] S. Roayaie, J. S. Frischer, S. H. Emre et al., "Long-term results with multimodal adjuvant therapy and liver transplantation for the treatment of hepatocellular carcinomas larger than 5 centimeters," Annals of Surgery, vol. 235, no. 4, pp. 533-539, 2002.

[19] N. M. Kneteman, J. Oberholzer, M. Al Saghier et al., "Sirolimus-based immunosuppression for liver transplantation in the presence of extended criteria for hepatocellular carcinoma," Liver Transplantation, vol. 10, no. 10, pp. 13011311, 2004.

[20] P. E. Majno, F. P. Sarasin, G. Mentha, and A. Hadengue, "Primary liver resection and salvage transplantation or primary liver transplantation in patients with single, small hepatocellular carcinoma and preserved liver function: an outcome-oriented decision analysis," Hepatology, vol. 31, no. 4, pp. 899-906, 2000.

[21] D. Fuks, S. Dokmak, V. Paradis, M. Diouf, F. Durand, and J. Belghiti, "Benefit of initial resection of hepatocellular carcinoma followed by transplantation in case of recurrence: an intention-to-treat analysis," Hepatology, vol. 55, no. 1, pp. 132-140, 2012.

[22] R. Adam, D. Azoulay, D. Castaing et al., "Liver resection as a bridge to transplantation for hepatocellular carcinoma on cirrhosis: a reasonable strategy?" Annals of Surgery, vol. 238, no. 4, pp. 508-519, 2003.

[23] P. E. Majno, R. Adam, H. Bismuth et al., "Influence of preoperative transarterial lipiodol chemoembolization on resection and transplantation for hepatocellular carcinoma in patients with cirrhosis," Annals of Surgery, vol. 226, no. 6, pp. 688-703, 1997.

[24] I. W. Graziadei, H. Sandmueller, P. Waldenberger et al., "Chemoembolization followed by liver transplantation for hepatocellular carcinoma impedes tumor progression while on the waiting list and leads to excellent outcome," Liver Transplantation, vol. 9, no. 6, pp. 557-563, 2003.

[25] F. Y. Yao, S. Breitenstein, C. E. Broelsch et al., "Does a patient qualify for liver transplantation after the down-staging of hepatocellular carcinoma?" Liver Transplantation, vol. 17, supplement 2, pp. S109-S116, 2011.

[26] O. Barakat, R. P. Wood, C. F. Ozaki et al., "Morphological features of advanced hepatocellular carcinoma as a predictor of downstaging and liver transplantation: an intention-totreat analysis," Liver Transplantation, vol. 16, no. 3, pp. 289299, 2010.

[27] F. Y. Yao, R. K. Kerlan Jr., R. Hirose et al., "Excellent outcome following down-staging of hepatocellular carcinoma prior to liver transplantation: an intention-to-treat analysis," Hepatology, vol. 48, no. 3, pp. 819-827, 2008.

[28] W. Y. Lau and E. C. H. Lai, "Salvage surgery following downstaging of unresectable hepatocellular carcinoma-a strategy to increase resectability," Annals of Surgical Oncology, vol. 14, no. 12, pp. 3301-3309, 2007.

[29] M. Ravaioli, G. L. Grazi, F. Piscaglia et al., "Liver transplantation for hepatocellular carcinoma: results of down-staging in patients initially outside the Milan selection criteria," 
American Journal of Transplantation, vol. 8, no. 12, pp. 25472557, 2008.

[30] R. J. Lewandowski, L. M. Kulik, A. Riaz et al., "A comparative analysis of transarterial downstaging for hepatocellular carcinoma: chemoembolization versus radioembolization," American Journal of Transplantation, vol. 9, no. 8, pp. 19201928, 2009.

[31] L. Kulik and M. Abecassis, "Living donor liver transplantation for hepatocellular carcinoma," Gastroenterology, vol. 127, supplement 1, pp. S277-S282, 2004.

[32] S. Di Sandro, A. O. Slim, A. Giacomoni et al., "Living donor liver transplantation for hepatocellular carcinoma: long-term results compared with deceased donor liver transplantation," Transplantation Proceedings, vol. 41, no. 4, pp. 1283-1285, 2009.

[33] K. Vakili, J. J. Pomposelli, Y. L. Cheah et al., "Living donor liver transplantation for hepatocellular carcinoma: Increased recurrence but improved survival," Liver Transplantation, vol. 15, no. 12, pp. 1861-1866, 2009.

[34] R. S. Mangus, J. A. Fridell, R. M. Vianna, A. B. Cooper, D. T. Jones, and A. J. Tector, "Use of the piggyback hepatectomy technique in liver transplant recipients with hepatocellular carcinoma," Transplantation, vol. 85, no. 10, pp. 1496-1499, 2008.

[35] P. A. Clavien, M. Lesurtel, P. M. Bossuyt et al., "Recommendations for liver transplantation for hepatocellular carcinoma: an international consensus conference report," The Lancet Oncology, vol. 13, pp. e11-e22, 2012.

[36] P. Bhangui, E. Vibert, P. Majno et al., "Intention-to-treat analysis of liver transplantation for hepatocellular carcinoma: living versus deceased donor transplantation," Hepatology, vol. 53, no. 5, pp. 1570-1579, 2011.

[37] J. Chen, K. Huang, J. Wu et al., "Survival after anatomic resection versus nonanatomic resection for hepatocellular carcinoma: a meta-analysis," Digestive Diseases and Sciences, vol. 56, no. 6, pp. 1626-1633, 2011.

[38] R. T. P. Poon, S. T. Fan, C. M. Lo et al., "Extended hepatic resection for hepatocellular carcinoma in patients with cirrhosis: is it justified?" Annals of Surgery, vol. 236, no. 5, pp. 602-611, 2002.

[39] H. Imamura, Y. Matsuyama, E. Tanaka et al., "Risk factors contributing to early and late phase intrahepatic recurrence of hepatocellular carcinoma after hepatectomy," Journal of Hepatology, vol. 38, no. 2, pp. 200-207, 2003.

[40] M. Samuel, P. K. Chow, E. Chan Shih-Yen, D. Machin, and K. C. Soo, "Neoadjuvant and adjuvant therapy for surgical resection of hepatocellular carcinoma," Cochrane Database of Systematic Reviews, no. 1, article CD001199, 2009.

[41] A. Abulkhir, P. Limongelli, A. J. Healey et al., "Preoperative portal vein embolization for major liver resection: a metaanalysis," Annals of Surgery, vol. 247, no. 1, pp. 49-57, 2008.

[42] K. P. Croome and M. H. Yamashita, "Laparoscopic vs open hepatic resection for benign and malignant tumors: an updated meta-analysis," Archives of Surgery, vol. 145, no. 11, pp. 1109-1118, 2010.

[43] T. Livraghi, F. Meloni, M. Di Stasi et al., "Sustained complete response and complications rates after radiofrequency ablation of very early hepatocellular carcinoma in cirrhosis: is resection still the treatment of choice?" Hepatology, vol. 47, no. 1, pp. 82-89, 2008.

[44] Y. K. Cho, J. K. Kim, M. Y. Kim, H. Rhim, and J. K. Han, "Systematic review of randomized trials for hepatocellular carcinoma treated with percutaneous ablation therapies," Hepatology, vol. 49, no. 2, pp. 453-459, 2009.
[45] R. Lencioni, "Loco-regional treatment of hepatocellular carcinoma," Hepatology, vol. 52, no. 2, pp. 762-773, 2010.

[46] S. Tanaka, M. Shimada, K. Shirabe et al., "Surgical radiofrequency ablation for treatment of hepatocellular carcinoma: an endoscopic or open approach," Hepato-Gastroenterology, vol. 56, no. 93, pp. 1169-1173, 2009.

[47] F. Di Benedetto, G. Tarantino, R. Montalti et al., "Laparoscopic radiofrequency ablation in the caudate lobe for hepatocellular carcinoma before liver transplantation," Journal of Laparoendoscopic \& Advanced Surgical Techniques, vol. 22, no. 4, pp. 400-402, 2012.

[48] Y. K. Cho, J. K. Kim, W. T. Kim, and J. W. Chung, "Hepatic resection versus radiofrequency ablation for very early stage hepatocellular carcinoma: a markov model analysis," Hepatology, vol. 51, no. 4, pp. 1284-1290, 2010.

[49] M. S. Chen, J. Q. Li, Y. Zheng et al., "A prospective randomized trial comparing percutaneous local ablative therapy and partial hepatectomy for small hepatocellular carcinoma," Annals of Surgery, vol. 243, no. 3, pp. 321-328, 2006.

[50] C. Cammà, V. Di Marco, A. Orlando et al., "Treatment of hepatocellular carcinoma in compensated cirrhosis with radiofrequency thermal ablation (RFTA): a prospective study," Journal of Hepatology, vol. 42, no. 4, pp. 535-540, 2005.

[51] R. Tateishi, S. Shiina, T. Teratani et al., "Percutaneous radiofrequency ablation for hepatocellular carcinoma: an analysis of 1000 cases," Cancer, vol. 103, no. 6, pp. 1201-1209, 2005.

[52] J. Huang, L. Yan, Z. Cheng et al., "A randomized trial comparing radiofrequency ablation and surgical resection for HCC conforming to the Milan criteria," Annals of Surgery, vol. 252, no. 6, pp. 903-912, 2010.

[53] M. Morimoto, K. Numata, M. Kondou, A. Nozaki, S. Morita, and K. Tanaka, "Midterm outcomes in patients with intermediate-sized hepatocellular carcinoma: a randomized controlled trial for determining the efficacy of radiofrequency ablation combined with transcatheter arterial chemoembolization," Cancer, vol. 116, no. 23, pp. 5452-5460, 2010.

[54] A. Veltri, P. Moretto, A. Doriguzzi, E. Pagano, G. Carrara, and G. Gandini, "Radiofrequency thermal ablation (RFA) after transarterial chemoembolization (TACE) as a combined therapy for unresectable non-early hepatocellular carcinoma (HCC)," European Radiology, vol. 16, no. 3, pp. 661-669, 2006.

[55] A. Forner, C. Ayuso, M. Varela et al., "Evaluation of tumor response after locoregional therapies in hepatocellular carcinoma: are response evaluation criteria in solid tumors reliable?" Cancer, vol. 115, no. 3, pp. 616-623, 2009.

[56] J. M. Llovet and J. Bruix, "Systematic review of randomized trials for unresectable hepatocellular carcinoma: chemoembolization improves survival," Hepatology, vol. 37, no. 2, pp. 429-442, 2003.

[57] J. L. Raoul, B. Sangro, A. Forner et al., "Evolving strategies for the management of intermediate-stage hepatocellular carcinoma: available evidence and expert opinion on the use of transarterial chemoembolization," Cancer Treatment Reviews, vol. 37, no. 3, pp. 212-220, 2011.

[58] M. Varela, M. I. Real, M. Burrel et al., "Chemoembolization of hepatocellular carcinoma with drug eluting beads: efficacy and doxorubicin pharmacokinetics," Journal of Hepatology, vol. 46, no. 3, pp. 474-481, 2007.

[59] J. Lammer, K. Malagari, T. Vogl et al., "Prospective randomized study of doxorubicin-eluting-bead embolization in the treatment of hepatocellular carcinoma: results of the PRECISION v study," CardioVascular and Interventional Radiology, vol. 33, no. 1, pp. 41-52, 2010. 
[60] G. Cabibbo, M. Enea, M. Attanasio, J. Bruix, A. Craxì, and C. Cammá, "A meta-analysis of survival rates of untreated patients in randomized clinical trials of hepatocellular carcinoma," Hepatology, vol. 51, no. 4, pp. 1274-1283, 2010.

[61] J. Luo, R. P. Guo, E. C. H. Lai et al., "Transarterial chemoembolization for unresectable hepatocellular carcinoma with portal vein tumor thrombosis: a prospective comparative study," Annals of Surgical Oncology, vol. 18, no. 2, pp. 413-420, 2011.

[62] L. M. Kulik, B. I. Carr, M. F. Mulcahy et al., "Safety and efficacy of $90 \mathrm{Y}$ radiotherapy for hepatocellular carcinoma with and without portal vein thrombosis," Hepatology, vol. 47, no. 1, pp. 71-81, 2008.

[63] L. M. Kulik, B. Atassi, L. van Holsbeeck et al., "Yttrium90 microspheres (TheraSphere) treatment of unresectable hepatocellular carcinoma: downstaging to resection, RFA and bridge to transplantation," Journal of Surgical Oncology, vol. 94, no. 7, pp. 572-586, 2006.

[64] B. Sangro, M. Iñarrairaegui, and J. I. Bilbao, "Radioembolization for hepatocellular carcinoma," Journal of Hepatology, vol. 56, no. 2, pp. 464-473, 2012.

[65] R. Salem, R. J. Lewandowski, L. Kulik et al., "Radioembolization results in longer time-to-progression and reduced toxicity compared with chemoembolization in patients with hepatocellular carcinoma," Gastroenterology, vol. 140, no. 2, pp. 497-507, 2011.

[66] J. K. Kang, M. S. Kim, C. K. Cho et al., "Stereotactic body radiation therapy for inoperable hepatocellular carcinoma as a local salvage treatment after incomplete transarterial chemoembolization," Cancer, vol. 118, no. 21, pp. 5424-5431, 2012.

[67] A. Takeda, M. Takahashi, E. Kunieda et al., "Hypofractionated stereotactic radiotherapy with and without transarterial chemoembolization for small hepatocellular carcinoma not eligible for other ablation therapies: preliminary results for efficacy and toxicity," Hepatology Research, vol. 38, no. 1, pp. 60-69, 2008.

[68] R. V. Tse, M. Hawkins, G. Lockwood et al., "Phase I study of individualized stereotactic body radiotherapy for hepatocellular carcinoma and intrahepatic cholangiocarcinoma," Journal of Clinical Oncology, vol. 26, no. 4, pp. 657-664, 2008.

[69] J. H. Kwon, S. H. Bae, J. Y. Kim et al., "Long-term effect of stereotactic body radiation therapy for primary hepatocellular carcinoma ineligible for local ablation therapy or surgical resection. Stereotactic radiotherapy for liver cancer," BMC Cancer, vol. 10, article 475, 2010.

[70] J. F. Perz, G. L. Armstrong, L. A. Farrington, Y. J. F. Hutin, and B. P. Bell, "The contributions of hepatitis B virus and hepatitis $\mathrm{C}$ virus infections to cirrhosis and primary liver cancer worldwide," Journal of Hepatology, vol. 45, no. 4, pp. 529-538, 2006.

[71] H. I. Yang, S. N. Lu, Y. F. Liaw et al., "Hepatitis B e antigen and the risk of hepatocellular carcinoma," The New England Journal of Medicine, vol. 347, pp. 168-174, 2002.

[72] H. L. Y. Chan, C. H. Tse, F. Mo et al., "High viral load and hepatitis B virus subgenotype Ce are associated with increased risk of hepatocellular carcinoma," Journal of Clinical Oncology, vol. 26, no. 2, pp. 177-182, 2008.

[73] Y. F. Liaw, J. J. Sung, W. C. Chow et al., "Lamivudine for patients with chronic hepatitis B and advanced liver disease," The New England Journal of Medicine, vol. 351, pp. 1521-1531, 2004.
[74] J. S. W. Wong, G. L. H. Wong, K. K. F. Tsoi et al., "Metaanalysis: the efficacy of anti-viral therapy in prevention of recurrence after curative treatment of chronic hepatitis Brelated hepatocellular carcinoma," Alimentary Pharmacology and Therapeutics, vol. 33, no. 10, pp. 1104-1112, 2011.

[75] S. Raimondi, S. Bruno, M. U. Mondelli, and P. Maisonneuve, "Hepatitis $\mathrm{C}$ virus genotype $\mathrm{lb}$ as a risk factor for hepatocellular carcinoma development: a meta-analysis," Journal of Hepatology, vol. 50, no. 6, pp. 1142-1154, 2009.

[76] L. T. Chen, M. F. Chen, L. A. Li et al., "Long-term results of a randomized, observation-controlled, phase III trial of adjuvant interferon Alfa-2b in hepatocellular carcinoma after curative resection," Annals of Surgery, vol. 255, no. 1, pp. 8-17, 2012.

[77] F. Sahin, R. Kannangai, O. Adegbola, J. Wang, G. Su, and M. Torbenson, "mTOR and P70 S6 kinase expression in primary liver neoplasms," Clinical Cancer Research, vol. 10, no. 24, pp. 8421-8425, 2004.

[78] A. Valdivieso, J. Bustamante, M. Gastaca et al., "Management of hepatocellular carcinoma recurrence after liver transplantation," Transplantation Proceedings, vol. 42, no. 2, pp. 660-662, 2010.

[79] J. M. Alamo, L. Barrera, M. D. Casado et al., "Efficacy, tolerance, and safety of mammalian target of rapamycin inhibitors as rescue immunosuppressants in liver transplantation," Transplantation Proceedings, vol. 41, no. 6, pp. 2181-2183, 2009.

[80] M. Vivarelli, A. Dazzi, M. Zanello et al., "Effect of different immunosuppressive schedules on recurrence-free survival after liver transplantation for hepatocellular carcinoma," Transplantation, vol. 89, no. 2, pp. 227-231, 2010.

[81] G. Söderdahl, L. Bäckman, H. Isoniemi et al., "A prospective, randomized, multi-centre trial of systemic adjuvant chemotherapy versus no additional treatment in liver transplantation for hepatocellular carcinoma," Transplant International, vol. 19, no. 4, pp. 288-294, 2006.

[82] H. Pokorny, M. Gnant, S. Rasoul-Rockenschaub et al., "Does additional doxorubicin chemotherapy improve outcome in patients with hepatocellular carcinoma treated by liver transplantation?" American Journal of Transplantation, vol. 5, no. 4, pp. 788-794, 2005.

[83] J. M. Llovet, S. Ricci, V. Mazzaferro et al., "Sorafenib in advanced hepatocellular carcinoma," The New England Journal of Medicine, vol. 359, no. 4, pp. 378-390, 2008.

[84] European Association for the Study of the Liver and European Organisation for Research and Treatment of Cancer, "EASLEORTC clinical practice guidelines: management of hepatocellular carcinoma," Journal of Hepatology, vol. 56, no. 4, pp. 908-943, 2012.

[85] R. Kim, G. El-Gazzaz, A. Tan et al., "Safety and feasibility of using sorafenib in recurrent hepatocellular carcinoma after orthotopic liver transplantation," Oncology, vol. 79, no. 1-2, pp. 62-66, 2010.

[86] M. Yeganeh, R. S. Finn, and S. Saab, "Apparent remission of a solitary metastatic pulmonary lesion in a liver transplant recipient treated with sorafenib," American Journal of Transplantation, vol. 9, no. 12, pp. 2851-2854, 2009.

[87] A. Villanueva and J. M. Llovet, "Targeted therapies for hepatocellular carcinoma," Gastroenterology, vol. 140, no. 5, pp. 1410-1426, 2011. 


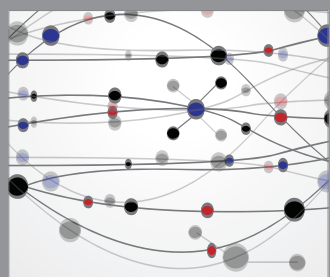

The Scientific World Journal
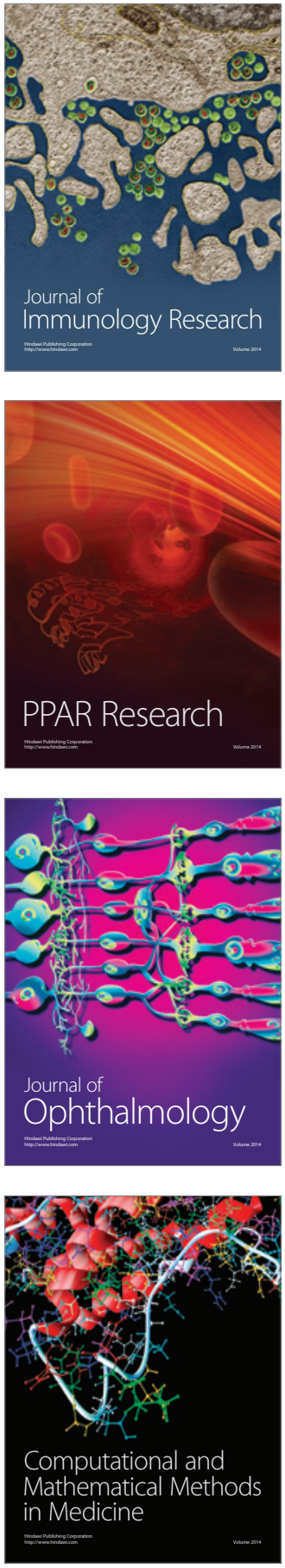

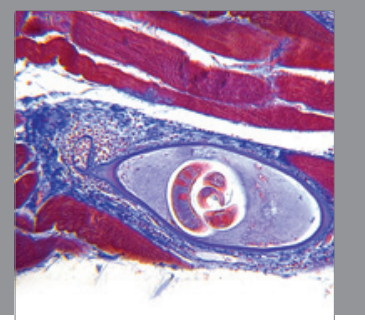

Gastroenterology

Research and Practice
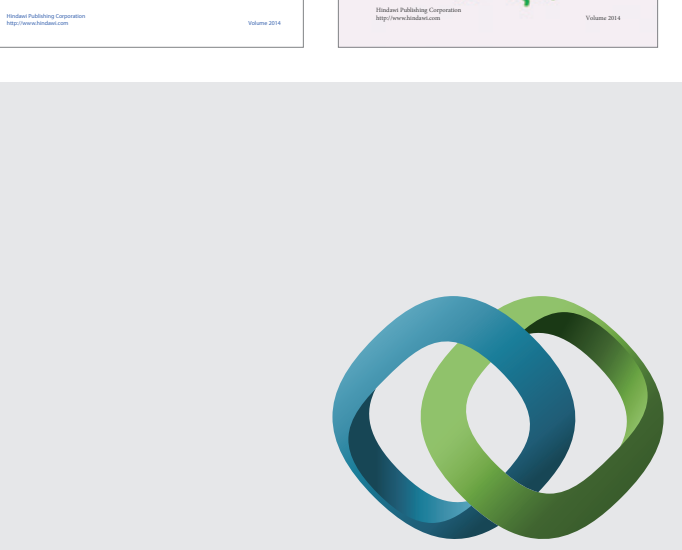

\section{Hindawi}

Submit your manuscripts at

http://www.hindawi.com
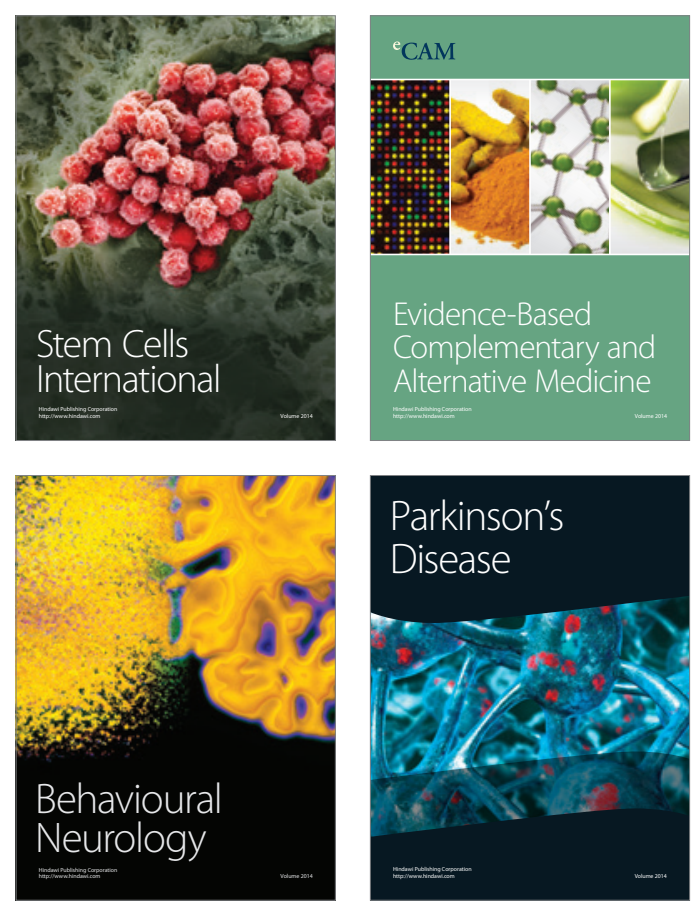

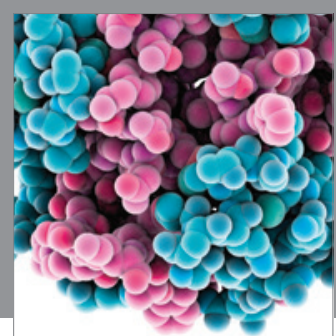

Journal of
Diabetes Research

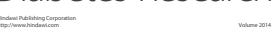

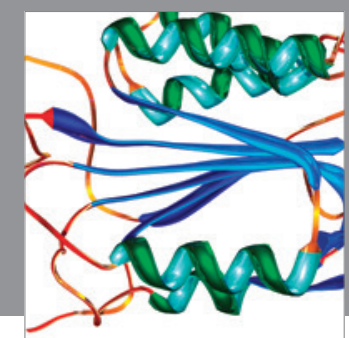

Disease Markers
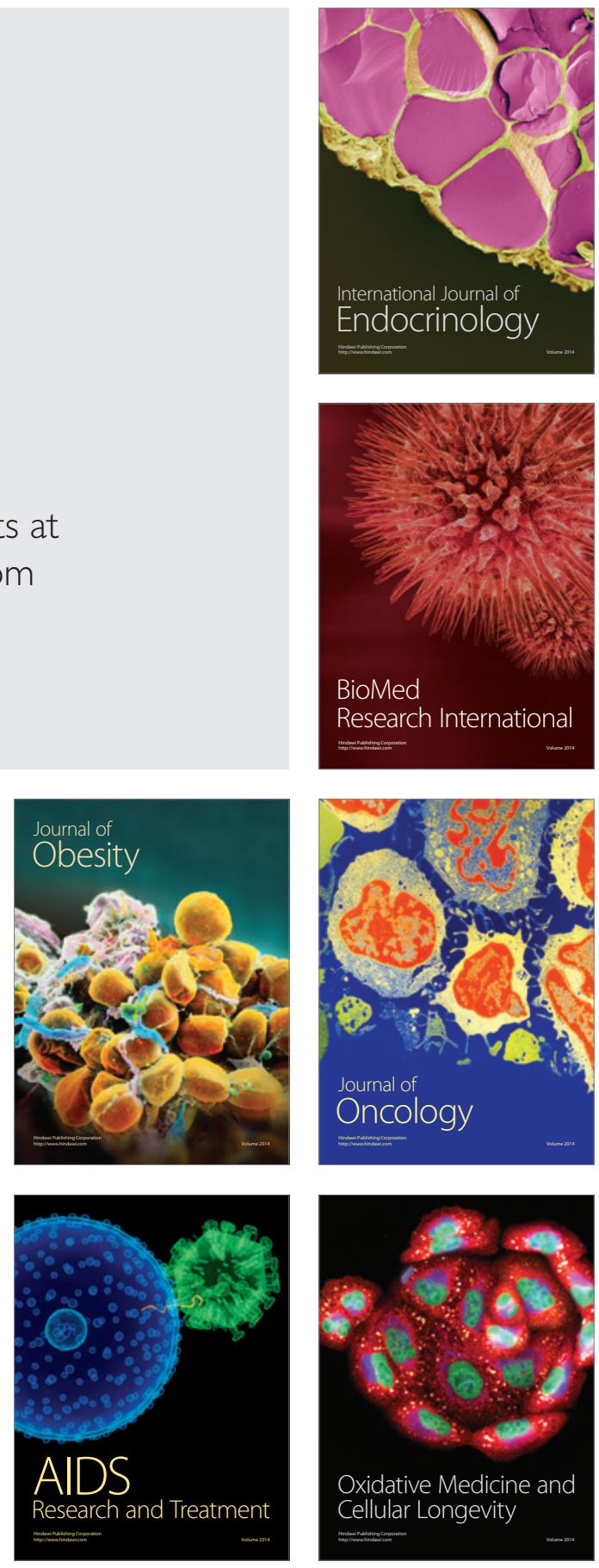